\title{
Association Of Serum Fibroblast Growth Factor 19 Levels With Arteriosclerosis Parameters Assessed By Arterial Stiffness And Atherogenic Index Of Plasma In Patients With Type 2 Diabetes
}

\section{Wangshu Liu}

Nantong City No 1 People's Hospital and Second Affiliated Hospital of Nantong University

Mengjie Tang

Nantong City No 1 People's Hospital and Second Affiliated Hospital of Nantong University

Tianli Xu

Nantong University Medical School

Jianbin Su

Nantong City No 1 People's Hospital and Second Affiliated Hospital of Nantong University xue-qin wang ( $\square$ wangxueqin108@163.com )

Feng Xu

Nantong City No 1 People's Hospital and Second Affiliated Hospital of Nantong University

Dongmei Zhang

Nantong City No 1 People's Hospital and Second Affiliated Hospital of Nantong University

Qing Zhu

Nantong City No 1 People's Hospital and Second Affiliated Hospital of Nantong University Jie Cao

Nantong City No 1 People's Hospital and Second Affiliated Hospital of Nantong University Hong Wang

Nantong City No 1 People's Hospital and Second Affiliated Hospital of Nantong University

\section{Research}

Keywords: Type 2 diabetes, FGF19, baPWV, atherogenic index of plasma

Posted Date: May 20th, 2020

DOI: https://doi.org/10.21203/rs.2.24087/v3

License: (c) (1) This work is licensed under a Creative Commons Attribution 4.0 International License. Read Full License 
Version of Record: A version of this preprint was published at Diabetology \& Metabolic Syndrome on May 20th, 2020. See the published version at https://doi.org/10.1186/s13098-020-00552-0. 


\section{Abstract}

Background The role of serum fibroblast growth factor 19 (FGF19) in arteriosclerosis is not well known. In the present study, we aimed to explore whether serum FGF19 levels were related to arteriosclerosis parameters, including arterial stiffness and atherogenic index of plasma (AIP), in patients with type 2 diabetes (T2D).

Methods A total of 200 patients with type 2 diabetes and 50 healthy controls were recruited for this study from Apr 2017 to Oct 2018. Serum FGF19 levels, arterial stiffness assessed by brachial ankle pulse wave velocity (baPWV), and AIP assessed by the triglyceride to high-density lipoprotein cholesterol (TG/HDL-c) ratio were measured in those subjects. In addition, other relevant clinical data were also collected.

Results Serum FGF19 levels in T2D patients were significantly lower than those in healthy controls $(p<$ 0.05). The arteriosclerosis parameters, including baPWV and AIP, significantly decreased across ascending tertiles of serum FGF19 levels (all $p$ for trend $<0.001$ ). Moreover, the baPWV and AIP were all inversely correlated with serum FGF19 levels ( $r=-0.351$ and -0.303 , respectively, $p<0.001$ ).

Furthermore, after adjusting for other clinical covariates by multiple linear regression analyses, the serum FGF19 levels were independently associated with baPWV $(\beta=-0.20, t=-2.23, p=0.029)$ and $\operatorname{AIP}(\beta=-$ $0.28, t=-2.66, p=0.010)$.

Conclusions The serum FGF19 levels were independently and inversely associated with baPWV and AIP, which indicate that serum FGF19 may have a protective role in atherosclerosis in patients with T2D.

\section{Background}

Cardiovascular disease (CAD) is a complication with high morbidity and mortality in patients with type 2 diabetes (T2D) [1]. Serum lipid deposition, leucocyte infiltration, and intimal thickening are three main steps in the development of atherosclerosis [2]. Dyslipidaemia has been considered a mediator and marker of CAD.

Arterial stiffness, as noninvasively measured by brachial-ankle pulse wave velocity (baPWV), is considered to be an independent predictor of CAD. Previous studies have reported that baPWV was closely correlated with the incidence of CAD and CAD-related mortality [3,4]. In addition, arterial stiffness is also a consequence of T2D and shared with the T2D in the pathogenesis of diabetic vascular complications $[5,6]$. BaPWV was also closely associated with glycosylated haemoglobin A1c (HbA1c), regardless of diabetes status [7]. A growing body of evidence has shown that the atherogenic index of plasma (AIP) is an important indicator of atherosclerosis and CAD [8-10]. As a new comprehensive lipid index, AIP is calculated from the plasma triglyceride (TG) value divided by the high-density lipoprotein cholesterol (HDL-c) value [11]. The AIP levels in T2D patients were significantly higher than those in healthy controls and may be account for the distribution of body fat and visceral fat area (VFA) [12]. 
Fibroblast growth factor 19 (FGF19), an endocrine hormone, is a member of the fibroblast growth factor (FGF) family. FGF19 is mainly produced in the intestinal epithelium and is expressed and functions in target tissues via FGF receptors (FGFR1, 2, 3, and 4) and $\beta$-Klotho. The main function of FGF19 is to regulate the synthesis of bile acid (BA) by acting on cholesterol 7a-hydroxylase (CYP7A1) [13,14]. As shown in our previous study, FGF19 participates in maintaining the balance of glucose and may protect against the dysfunction of $\beta$-cells [15]. The serum FGF19 levels in CAD subjects are lower than those in subjects without CAD and are correlated with the presence and severity of CAD [16]. However, few studies revealed the associations of FGF19 with arteriosclerosis indices, such as baPWV and AIP.

The aim of the present study was to investigate whether serum FGF19 levels were related to the arteriosclerosis parameters, including baPWV and AIP, in patients with type 2 diabetes. We found that serum FGF19 may have a protective role in atherosclerosis in patients with T2D.

\section{Methods}

\section{Study design and population}

We recruited 200 patients with type 2 diabetes who visited and were followed up in the Endocrinology Department at Affiliated Hospital 2 of Nantong University from April 2017 to October 2018, and 50 healthy controls were selected from the Department of Physical Examination Center during the same period. The inclusion criteria for T2D patients were age from 20 to 75 years and a diagnosis of T2D [17]. The inclusion criteria for controls were age from 20 to 75 years and having a fasting plasma glucose (FPG) level $<6.1 \mathrm{mmol} / \mathrm{L}$ and a 2-h plasma glucose (2hPG) level $<7.8 \mathrm{mmol} / \mathrm{L}$ [17]. Subjects who had type 1 diabetes, hyperthyroidism or hypothyroidism, severe hepatic disease such as chronic viral hepatitis, chronic renal insufficiency with estimated glomerular filtration rate $<90 \mathrm{ml} / \mathrm{min} / 1.73 \mathrm{~m}^{2}$, previous cancer, acute diabetic complications such as diabetic ketoacidosis, and current treatment with systemic corticosteroids were also excluded. The brachial-ankle pulse wave velocity (baPWV) was determined by a non-invasive vascular screening device (BP-203RPE III device, Omron Healthcare, Kyoto, Japan) while the patient was in the supine position and after a rest of at least five minutes. All participants provided informed consent, and the study protocol was approved by the Ethics Committee of Affiliated Hospital 2 of Nantong University and First People's Hospital of Nantong City.

\section{Anthropometric measures and calculation}

All participants finished a questionnaire collecting demographic and anthropometric data under the supervision of experienced investigators after fasting for 8 hours. Hypertension was tested at least 3 times and was defined as a systolic arterial blood pressure $\geq 140 \mathrm{mmHg}$ and/or diastolic blood pressure $\geq 90 \mathrm{mmHg}$ after at least $0.5 \mathrm{~h}$ of rest. Hepatic steatosis on ultrasound was used to establish the diagnosis of non-alcoholic fatty liver disease (NAFLD). The calculation of the homeostatic model assessment for insulin sensitivity $\left(\mathrm{IS}_{\text {HOMA-cp }}\right)$ [18], Body mass index (BMI), AIP and NAFLD fibrosis score (NFS) was as follows: $I_{\text {HOMA-cp }}=22.5 /$ (glucose $\times$ C-peptide in fasting status); $\mathrm{BMI}=$ the weight $/$ the 
height squared; AIP = TG/HDL-c; NFS $=-1.675+0.037 \times$ age $($ years $)+0.094 \times \mathrm{BMI}\left(\mathrm{kg} / \mathrm{m}^{2}\right)+1.13 \times$ $\mathrm{IFG} /$ diabetes $(\mathrm{yes}=1, \mathrm{no}=0)+0.99 \times$ AST $/$ ALT ratio $-0.013 \times$ platelet $\left(\times 10^{9} / \mathrm{l}\right)-0.66 \times$ albumin $(\mathrm{g} / \mathrm{dl})$ [15].

\section{Serum biochemical indicators}

Peripheral blood samples were collected after $>12$ hours of fasting. TG was measured using colorimetry, total cholesterol (TC) was measured with the cholesterol oxidase method, low-density lipoprotein cholesterol (LDL-c) was determined with the selective melt method and HDL-c was measured using the modified enzyme method. All levels were determined by an automated biochemical instrument (Model 7600, Hitachi). lonic exchange HPLC (IE-HPLC) in the D-10 haemoglobin analysis system (Bio-Rad) was used to determine the $\mathrm{HbA} 1 \mathrm{c}$ level. Insulin levels, fasting blood glucose concentrations and 2-h blood glucose concentrations were measured by laboratory procedures $[16,17]$. All blood samples were stored at $-80^{\circ} \mathrm{C}$. Serum FGF19 levels were measured by sandwich ELISA (FGF19 Quantikine ${ }^{8}$ DF1900; R\&D Systems, Minneapolis, MN, USA). The intra- and inter-assay coefficients of variation were $4.3 \%$ and $5.6 \%$, respectively.

\section{Statistical analysis}

SPSS 25.0 (Inc., Chicago, IL) statistical software was used. Normally distributed data are expressed as the mean \pm SD. The Kruskal-Wallis test was performed to assess the variables' distributions. If the distributions of the data were skewed, a natural logarithm transformation (In) was applied to achieve a normal distribution for further analysis, such as InFGF19 and InAIP. The differences in continuous variables between the FGF19 tertiles were compared by one-way analysis of variance (ANOVA), and the categorical variables between the three groups were compared by the chi-square test. Spearman's bivariate correlation analysis was used to analyse the correlations of InFGF19 with baPWV and InFGF19 with InAIP. Considering that age, gender, duration, BMI, hypertension, diabetic treatment and NAFLD may affect the values of baPWV and AIP, two partial correlation analyses were conducted to explore the associations of InFGF19 with baPWV and InFGF19 with InAIP, adjusting for age, gender, duration, BMI, hypertension, diabetic treatment and NAFLD. Furthermore, two multiple stepwise linear regression analyses were used to explore the independent factors related to FGF19 levels for baPWV and to FGF19 levels for AIP. A $p$ value $<0.05$ was defined as significant.

\section{Results}

\section{Basic characteristics}

The characteristics of the participants are shown in Table 1. Compared with healthy controls, T2D patients were older and had higher BMI, systolic pressure (SBP), diastolic blood pressure (DBP), fasting plasma glucose (FPG) and 2-hour postprandial blood glucose (2hPG) (all $p<0.01$ ). The FGF19 concentrations were significantly lower in the T2D group than the control group. The mean FGF19 levels for the whole T2D group were 138.18 (66.46-181.31), and the tertiles were T1 (< 82.6), T2 (82.6-149.6), 
and T3 (> 149.6). According to the examination results, prominent differences were found for AIP and baPWV from T1 to T3 (all $p$ for trend < 0.001). Moreover, from T1 to T3, the number of T2D patients with hypertension decreased significantly ( $p$ for trend $<0.05$ ), but no significant difference was found in the number of T2D patients with NAFLD and the NFS values. The mean NFS for T2D participants was $-23.71 \pm 2.65$, which indicates that for most T2D patients with NAFLD, the negative predictive value for fibrosis was $93 \%$ [19].

\section{Relationships between InFGF19 and arteriosclerosis parameters}

Spearman's bivariate correlation analysis was conducted to assess the relationships between InFGF19 levels and arteriosclerosis parameters in the T2D group. As shown in Fig 1 and Fig 2, the serum InFGF19 levels were significantly and negatively related to baPWV and InAIP $(r=-0.351, p<0.001 ; r=-0.321, p<$ 0.001 , respectively). Interestingly, the associations all still existed after adjusting for age, gender, duration, $\mathrm{BMI}$, hypertension, diabetic treatment and NAFLD $(r=-0.321, p<0.001 ; r=-0.256, p<0.001$, respectively).

\section{Multiple linear regression models displayed independent associations of InFGF19 with arteriosclerosis parameters}

Table 2 shows the associations of InFGF19 with arteriosclerosis parameters (baPWV and AIP) based on multiple linear regression analyses. In the basal unadjusted model 0 , InFGF19 was significantly associated with baPWV $\left(\beta=-0.37, t=-5.57, p<0.001\right.$, adjusted $\left.R^{2}=0.135\right)$ and $\operatorname{lnAIP}(\beta=-0.30, t=-$ $4.46, p<0.001$, adjusted $\left.R^{2}=0.091\right)$. After gradually adding the other clinical covariates in each model, we observed a gradual increase in the adjusted $R^{2}$. In the fully adjusted model 3, InFGF19 was still independently associated with baPWV $\left(\beta=-0.20, t=-2.23, p=0.029\right.$, adjusted $\left.R^{2}=0.599\right)$ and InAIP ( $\beta$ $=-0.28, t=-2.26, p=0.010$, adjusted $\left.R^{2}=0.491\right)$. As a result, the serum FGF19 levels were independently and inversely associated with baPWV and AIP in patients with T2D.

\section{Discussion}

We conducted an observational study to compare the serum FGF19 levels between the patients with T2D and healthy controls and to analyse the association of serum FGF19 levels with baPWV and FGF19 levels with AIP in patients with T2D. The main findings of our study are as follows: first, serum FGF19 levels of T2D patients were significantly lower than those in healthy controls; second, arteriosclerosis parameters, including baPWV and AIP, significantly decreased across ascending tertiles of serum FGF19 levels in patients with T2D; and third, after adjusting for other clinical covariates, the serum FGF19 levels were independently and inversely associated with baPWV in patients with T2D.

Evidence from previous studies has shown that FGF19 participates in the synthesis of BA, the balance of glucose metabolism, and the reduction of weight in mice [20-23]. In accordance with our data, J. Zhang et al. showed that serum FGF19 levels were significantly lower in normal glucose tolerance (NGT) subjects 
than in isolated-impaired glucose tolerance (I-IGT) subjects and isolated-impaired fasting glucose (I-IFG) participants based on glucose effectiveness (GE) and hepatic glucose production (HGP) [24]. Meanwhile, in T2D patients with MetS, serum FGF19 levels were significantly lower than they were in other T2D patients. Moreover, FGF19 levels were significantly negatively related to AIP and TG in T2D patients with MetS [25]. In a study of 315 Chinese patients with suspected or established CAD, the serum FGF19 level was an independent predictor of the Gensini score, which represents the presence and severity of CAD [16]. In our study, the FGF19 levels in the T2D group decreased significantly compared with those in the control group. In the T2D group, InFGF19 levels were negatively related to baPWV and AIP values.

Arterial stiffness was considered to be an independent predictor of atherosclerotic diseases and can be measured by baPWV, a simple, noninvasive and convenient tool [26,27]. Previous studies revealed that baPWV was broadly used and generally accepted in China [28]. BaPWV was associated with blood pressure, diabetes status [29], HbA1c [7], inflammation [30] and obesity [31]. We considered baPWV as a risk factor for CVD and investigated its association with serum FGF19 levels. Finally, our study found that in the fully adjusted model 3 , InFGF19 was still independently associated with baPWV $(\beta=-0.20, t=-$ 2.23, $p=0.029$, adjusted $\left.R^{2}=0.599\right)$.

AIP is a main marker for the presence of atherosclerosis and CAD [32,33]. In postmenopausal women with CAD, the values of AIP were higher than those in the control group. After multivariate logistic regression analysis, AIP was shown to be independently related to CAD, which indicated that AIP was a significant marker for the incidence of CAD [34]. Gaojun Cai et al found that in the Han Chinese population, AIP was a predictor for the incidence of CAD [33]. The patients with diabetic neuropathy and MetS had significantly higher AIP levels than their counterparts [35]. In our study, the levels of AIP in the T2D group were higher than those in the controls, and AIP was independently associated with the serum FGF19 levels in T2D patients. In agreement with our study, P. Song et al. revealed that the AIP was obviously higher in T2D participants than in non-T2D participants [12]. However, more research should be conducted to analyse the relationship between FGF19 levels and arterial lesions.

Several possible mechanisms may explain the link between FGF19 levels and atherosclerosis in T2D patients. FGF19 regulates glucolipid homeostasis and nutrient metabolism via the FGFR4- $\beta$-Klotho complex. In rodent studies, FXR-/- mice showed a pro-atherogenetic lipoprotein profile and defects in the formation of any detectable plaques associated with a high-fat (HF) diet. FXR agonists protect against the formation of aortic plaques in murine models that have a pro-atherogenetic lipoprotein profile and accelerated atherosclerosis [36]. In hepatic FXR-knockout and FXR-knockdown mice, the reconstitution of FXR expression upregulated the transport of cholesterol. Consistent with its role of phosphorylating FXR, the nonreceptor tyrosine kinase Src regulated the formation of cholesterol and ameliorated arterial lesions. Therefore, the phosphorylation of hepatic FXR induced Src via FGF15/19 and then played a role in the balance of cholesterol homeostasis, preventing the formation of atherosclerosis [37]. In transgenic human apolipoprotein(a) (APOA) mice (tg-APO mice), the FGF19 influence APOA biosynthesis by attenuating transfection of primary hepatocytes with siRNA against the FGFR4 [38]. Moreover, Mei Zhou, R. et al. revealed that NGM282, an FGF19 analogue, regulated cholesterol in mice by activating MEK1 and 
reduced atherosclerosis in Apoe-/- mice with dyslipidaemia. Furthermore, the HDL-c levels of healthy volunteers improved after the administration of NGM282 for a week [39]. Therefore, our research revealed that baPWV and AIP were all independently associated with FGF19 levels after adjusting for other clinical covariates via multiple linear regression analysis, which indicates that decreased FGF19 may be accompanied by several unfavourable metabolic alterations and can be implicated in increased arterial stiffness.

This study had several limitations that should be addressed. First, this study had a cross-sectional design based on a small sample size and could not explain any causal connection between the decreased serum FGF19 level and increased risk of atherosclerosis and CAD as assessed by baPWV and AIP. Second, our research was a single-centre study conducted among Chinese participants, and the generalizability of our data needs to be assessed. Third, the influence of diabetic and hypertensive treatment on the formation of atherosclerosis is unknown, and the influence of medication history on baPWV and AIP values has not been determined. However, InFGF19 levels were closely related to baPWV and AIP, even after adjusting for age, gender, duration, BMI, hypertension, diabetic treatment and NAFLD. Meanwhile, baPWV was not measured among controls, but the present research showed that diabetes was significantly correlated with an increased risk of baPWV compared with normal glucose [29]. Moreover, other confounding factors affecting baPWV, such as alcohol consumption, smoking status and health behaviour, were not completely assessed at the beginning of this study. Finally, the relationships between FGF19 levels and CAD measured by other vascular markers, such as carotid intima-media thickness (CIMT) or flowmediated dilatation (FMD), were not assessed. All limitations were fully considered when selecting the appropriate statistical approach. Therefore, larger, prospective follow-up studies need to be conducted to better investigate the correlation between FGF19 levels and the risk of CAD in T2D patients.

\section{Conclusions}

The serum FGF19 levels were independently and inversely associated with baPWV and AIP, which indicate that serum FGF19 may have a protective role in atherosclerosis in patients with T2D.

\section{Abbreviations}

CAD: cardiovascular disease, T2D: type 2 diabetes, baPWV: brachial-ankle pulse wave velocity, CN: controls, BMI: body mass index, SBP: systolic blood pressure, DBP: diastolic blood pressure, FPG: fasting plasma glucose, 2hPG: 2-hour postprandial blood glucose, HbA1c: glycosylated haemoglobin A1c, BA:

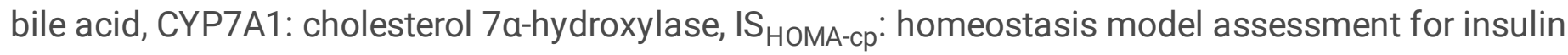
sensitivity calculated using glucose and C-peptide in fasting status, TC: total cholesterol, TG: triglyceride, HDL-c: high-density lipoprotein cholesterol, LDL-c: low-density lipoprotein cholesterol, FGF19: fibroblast growth factor 19, AIP: atherogenic index of plasma, OGTT: oral glucose tolerance test, NGT: normal glucose tolerance; IIGT: isolated-impaired glucose tolerance; I-IFG; isolated-impaired fasting glucose; GE: glucose effectiveness, HGP: hepatic glucose production, MetS: metabolic syndrome, HF: high-fat, NAFLD: 
non-alcoholic fatty liver disease, NFS: NAFLD fibrosis score, CIMT: carotid intima-media thickness, FMD: flow-mediated dilatation, APOA: apolipoprotein(a) .

\section{Declarations}

\section{Authors' contributions}

WL, XW and TX participated in the design of the study, data collection, analysis of the data, and drafting of the manuscript. JS and MT conceived of the study, participated in its design and revised the manuscript. MT and TX participated in the analysis of the data and revised the manuscript. MT, FX, DZ, QZ, JC and HW participated in data collection. All authors read and approved the final manuscript.

\section{Author details}

1 Department of Endocrinology, Affiliated Hospital 2 of Nantong University and First People's Hospital of Nantong City, No. 6 North Hai-er-xiang Road, Nantong 226001, China. 2 Department of Clinical Laboratory, Affiliated Hospital 2 of Nantong University and First People's Hospital of Nantong City, No. 6 North Hai-er-xiang Road, Nantong 226001, China. 3 Student of Medical College of Nantong University, No. 19 Qi-xiu Road, Nantong 226001, China.

\section{Acknowledgements}

Not applicable.

\section{Competing interests}

The authors declare that they have no competing interests.

\section{Availability of data and materials}

The current data are available to all interested researchers upon reasonable request. Requests for access to data should be made to the principal investigators of the study.

\section{Consent for publication}

Not applicable.

\section{Ethics approval and consent to participate}

The study was approved by the institutional review board of Affiliated Hospital 2 of Nantong University and First People's Hospital of Nantong City, and written informed consent was obtained from all participants.

\section{Funding}


No funding.

\section{References}

1. Mathers CD, Loncar D. Projections of global mortality and burden of disease from 2002 to 2030. PLoS Med 2006; 3:e442

2. Crowther MA. Pathogenesis of atherosclerosis. Hematology Am Soc Hematol Educ Program 2005:436-441

3. Kaess BM, Rong J, Larson MG, Hamburg NM, Vita JA, et al. Aortic stiffness, blood pressure progression, and incident hypertension. JAMA 2012; 308:875-881

4. Cavalcante JL, Lima JA, Redheuil A, Al-Mallah MH. Aortic stiffness: current understanding and future directions. J Am Coll Cardiol 2011; 57:1511-1522

5. Chen Q, Chiheb S, Fysekidis M, Jaber Y, Brahimi M, et al. Arterial stiffness is elevated in normotensive type 2 diabetic patients with peripheral neuropathy. Nutr Metab Cardiovasc Dis 2015; 25:1041-1049

6. Xu M, Huang Y, Xie L, Peng K, Ding L, et al. Diabetes and Risk of Arterial Stiffness: A Mendelian Randomization Analysis. Diabetes 2016; 65:1731-1740

7. Wen J, Hu F, Yang Q. Comparison of Hemoglobin Alc, Glycated Albumin and Fasting Plasma Glucose for Prediction of Arterial Stiffness in Chinese Adults. Diabetes Metab Syndr Obes 2020; 13:65-70

8. Chang Y, Li Y, Guo X, Dai D, Sun Y. The Association of Ideal Cardiovascular Health and Atherogenic Index of Plasma in Rural Population: A Cross-Sectional Study from Northeast China. Int J Environ Res Public Health 2016; 13

9. Zhan Y, Xu T, Tan X. Two parameters reflect lipid-driven inflammatory state in acute coronary syndrome: atherogenic index of plasma, neutrophil-lymphocyte ratio. BMC Cardiovasc Disord 2016; $16: 96$

10. Niroumand S, Khajedaluee M, Khadem-Rezaiyan M, Abrishami M, Juya M, et al. Atherogenic Index of Plasma (AIP): A marker of cardiovascular disease. Med J Islam Repub Iran 2015; 29:240

11. Dobiasova $M$, Frohlich J. The plasma parameter log (TG/HDL-C) as an atherogenic index: correlation with lipoprotein particle size and esterification rate in apoB-lipoprotein-depleted plasma (FER(HDL)). Clin Biochem 2001; 34:583-588

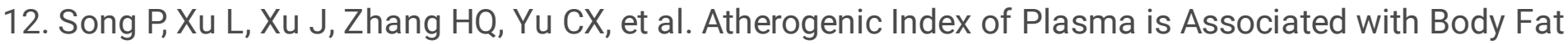
Level in Type 2 Diabetes Mellitus Patients. Curr Vasc Pharmacol 2018; 16:589-595

13. Kliewer SA, Mangelsdorf DJ. Bile Acids as Hormones: The FXR-FGF15/19 Pathway. Dig Dis 2015; 33:327-331

14. Degirolamo C, Sabba C, Moschetta A. Therapeutic potential of the endocrine fibroblast growth factors FGF19, FGF21 and FGF23. Nat Rev Drug Discov 2016; 15:51-69

15. Tang MJ, Su JB, Xu TL, Wang XQ, Zhang DM, et al. Serum fibroblast growth factor 19 and endogenous islet beta cell function in type 2 diabetic patients. Diabetol Metab Syndr 2019; 11:79 
16. Hao Y, Zhou J, Zhou M, Ma X, Lu Z, et al. Serum levels of fibroblast growth factor 19 are inversely associated with coronary artery disease in chinese individuals. PLoS One 2013; 8:e72345

17. Alberti KG, Zimmet PZ. Definition, diagnosis and classification of diabetes mellitus and its complications. Part 1: diagnosis and classification of diabetes mellitus provisional report of a WHO consultation. Diabet Med 1998; 15:539-553

18. Radaelli T, Farrell KA, Huston-Presley L, Amini SB, Kirwan JP, et al. Estimates of insulin sensitivity using glucose and C-Peptide from the hyperglycemia and adverse pregnancy outcome glucose tolerance test. Diabetes Care 2010; 33:490-494

19. Angulo P, Hui JM, Marchesini G, Bugianesi E, George J, et al. The NAFLD fibrosis score: a noninvasive system that identifies liver fibrosis in patients with NAFLD. Hepatology 2007; 45:846-854

20. Zhou M, Learned RM, Rossi SJ, DePaoli AM, Tian H, et al. Engineered FGF19 eliminates bile acid toxicity and lipotoxicity leading to resolution of steatohepatitis and fibrosis in mice. Hepatol Commun 2017; 1:1024-1042

21. Tomlinson E, Fu L, John L, Hultgren B, Huang X, et al. Transgenic mice expressing human fibroblast growth factor-19 display increased metabolic rate and decreased adiposity. Endocrinology 2002; 143:1741-1747

22. Massafra V, Milona A, Vos HR, Burgering BM, van Mil SW. Quantitative liver proteomics identifies FGF19 targets that couple metabolism and proliferation. PLoS One 2017; 12:e0171185

23. Hansen AMK, Vienberg SG, Lykkegaard K, Zhao X, Tingqing G, et al. Differential receptor selectivity of the FGF15/FGF19 orthologues determines distinct metabolic activities in $\mathrm{db} / \mathrm{db}$ mice. Biochem J 2018; 475:2985-2996

24. Zhang J, Li H, Bai N, Xu Y, Song Q, et al. Decrease of FGF19 contributes to the increase of fasting glucose in human in an insulin-independent manner. J Endocrinol Invest 2019; 42:1019-1027

25. Barutcuoglu B, Basol G, Cakir Y, Cetinkalp S, Parildar Z, et al. Fibroblast growth factor-19 levels in type 2 diabetic patients with metabolic syndrome. Ann Clin Lab Sci 2011; 41:390-396

26. Ninomiya T, Kojima I, Doi Y, Fukuhara M, Hirakawa Y, et al. Brachial-ankle pulse wave velocity predicts the development of cardiovascular disease in a general Japanese population: the Hisayama Study. J Hypertens 2013; 31:477-483; discussion 483

27. Boutouyrie P, Tropeano Al, Asmar R, Gautier I, Benetos A, et al. Aortic stiffness is an independent predictor of primary coronary events in hypertensive patients: a longitudinal study. Hypertension 2002; 39:10-15

28. Lu YC, Lyu P, Zhu HY, Xu DX, Tahir S, et al. Brachial-ankle pulse wave velocity compared with mean arterial pressure and pulse pressure in risk stratification in a Chinese population. J Hypertens 2018; 36:528-536

29. Dai X, Yang Y, Cheng GL, Jia J, Fan FF, et al. Higher blood pressure increases arterial stiffness modified by blood glucose levels in a Chinese community-based study. Diabetes Metab Syndr Obes $2019 ; 12: 901-911$ 
30. Sun L, Ning C, Liu J, Yao T, Zhang L, et al. The association between cumulative C-reactive protein and brachial-ankle pulse wave velocity. Aging Clin Exp Res 2019;

31. Choi HS, Cho YH, Lee SY, Park EJ, Kim YJ, et al. Association between new anthropometric parameters and arterial stiffness based on brachial-ankle pulse wave velocity. Diabetes Metab Syndr Obes 2019; 12:1727-1733

32. Nwagha UI, Ikekpeazu EJ, Ejezie FE, Neboh EE, Maduka IC. Atherogenic index of plasma as useful predictor of cardiovascular risk among postmenopausal women in Enugu, Nigeria. Afr Health Sci 2010; 10:248-252

33. Cai G, Shi G, Xue S, Lu W. The atherogenic index of plasma is a strong and independent predictor for coronary artery disease in the Chinese Han population. Medicine (Baltimore) 2017; 96:e8058

34. Wu TT, Gao Y, Zheng YY, Ma YT, Xie X. Atherogenic index of plasma (AIP): a novel predictive indicator for the coronary artery disease in postmenopausal women. Lipids Health Dis 2018; 17:197

35. Li Z, Huang Q, Sun L, Bao T, Dai Z. Atherogenic Index in Type 2 Diabetes and Its Relationship with Chronic Microvascular Complications. Int J Endocrinol 2018; 2018:1765835

36. Mencarelli A, Fiorucci S. FXR an emerging therapeutic target for the treatment of atherosclerosis. J Cell Mol Med 2010; 14:79-92

37. Byun S, Jung H, Chen J, Kim YC, Kim DH, et al. Phosphorylation of hepatic farnesoid X receptor by FGF19 signaling-activated Src maintains cholesterol levels and protects from atherosclerosis. J Biol Chem 2019; 294:8732-8744

38. Chennamsetty I, Claudel T, Kostner KM, Trauner M, Kostner GM. FGF19 signaling cascade suppresses APOA gene expression. Arterioscler Thromb Vasc Biol 2012; 32:1220-1227

39. Zhou M, Learned RM, Rossi SJ, Tian H, DePaoli AM, et al. Therapeutic FGF19 promotes HDL biogenesis and transhepatic cholesterol efflux to prevent atherosclerosis. J Lipid Res 2019; 60:550565

\section{Tables}

Table 1 Characteristics of the study participants 


\begin{tabular}{|c|c|c|c|c|c|c|}
\hline \multirow[t]{2}{*}{ Variable } & \multirow[t]{2}{*}{ Controls } & \multicolumn{4}{|l|}{$\mathrm{T} 2 \mathrm{D}$} & \multirow{2}{*}{$\begin{array}{l}p \text { for } \\
\text { trend }\end{array}$} \\
\hline & & Total & T1 & $\mathrm{T} 2$ & T3 & \\
\hline FGF19 (pg/mL) & $\begin{array}{l}232.3(122.8- \\
280.8)\end{array}$ & $\begin{array}{l}138.2(66.5- \\
181.3) * *\end{array}$ & $<82.6$ & $82.6-149.6$ & $>149.6$ & \\
\hline N (male\%) & $50(19.6)$ & $200(60.5)^{* *}$ & $67(62.7)$ & $67(53.7)$ & $66(65.2)$ & 0.776 \\
\hline Duration(months) & $\mathrm{N}$ & $\begin{array}{l}49.23(1.0- \\
72.0)\end{array}$ & $\begin{array}{l}51.38(2.0- \\
96.0)\end{array}$ & $\begin{array}{l}61.22(1.0- \\
96.0)\end{array}$ & $\begin{array}{l}34.87(1.0- \\
36.25)\end{array}$ & 0.185 \\
\hline Age (years) & $35.3 \pm 10.9$ & $52.4 \pm 10.7^{* *}$ & $52.1 \pm 11.2$ & $52.3 \pm 10.2$ & $53.0 \pm 10.8$ & 0.626 \\
\hline BMI (kg/m2) & $22.5 \pm 2.7$ & $25.8 \pm 3.6^{* *}$ & $26.3 \pm 3.5$ & $26.0 \pm 3.1$ & $25.2 \pm 4.1$ & 0.061 \\
\hline SBP (mmHg) & $109.8 \pm 7.1$ & $136.2 \pm 16.4^{* *}$ & $134.6 \pm 16.8$ & $136.7 \pm 15.0$ & $137.5 \pm 17.4$ & 0.300 \\
\hline DBP (mmHg) & $76.0 \pm 9.5$ & $81.9 \pm 11.2^{* *}$ & $80.8 \pm 10.8$ & $81.7 \pm 9.9$ & $83.3 \pm 12.7$ & 0.211 \\
\hline \multicolumn{7}{|l|}{$\begin{array}{l}\text { Antidiabetic } \\
\text { treatment }\end{array}$} \\
\hline $\begin{array}{l}\text { Lifestyle intervention } \\
\text { alone, } \mathrm{n}(\%)\end{array}$ & $\mathrm{N}$ & $2(1.0)$ & 0 & $1(1.5)$ & $1(1.5)$ & 0.252 \\
\hline $\begin{array}{l}\text { Insulin treatments, } \mathrm{n} \\
(\%)\end{array}$ & $\mathrm{N}$ & $136(68.0)$ & $43(64.2)$ & $49(73.1)$ & $44(66.7)$ & 0.250 \\
\hline $\begin{array}{l}\text { Insulin- } \\
\text { secretagogues, n (\%) }\end{array}$ & $\mathrm{N}$ & $87(43.5)$ & $27(40.3)$ & $32(47.8)$ & $28(42.4)$ & 0.290 \\
\hline $\begin{array}{l}\text { Insulin-sensitisers, } \mathrm{n} \\
(\%)\end{array}$ & $\mathrm{N}$ & $24(12.0)$ & $6(9.0)$ & $10(14.9)$ & $8(12.1)$ & 0.511 \\
\hline Hypertension, n (\%) & $\mathrm{N}$ & $89(44.5)$ & $33(49.3)$ & $27(40.3)$ & $29(43.9)$ & $0.047^{\#}$ \\
\hline Statins, n (\%) & $\mathrm{N}$ & $17(8.5)$ & $10(14.9)$ & $3(4.5)$ & $4(6.1)$ & 0.711 \\
\hline NAFLD, n (\%) & $\mathrm{N}$ & $89(44.5)$ & $35(52.2)$ & $25(37.3)$ & $29(43.9)$ & 0.264 \\
\hline NFS & $\mathrm{N}$ & $-23.71 \pm 2.65$ & $-24.25 \pm 2.61$ & $-23.20 \pm 2.69$ & $-23.71 \pm 2.59$ & 0.264 \\
\hline FPG (mmol/L) & $4.63 \pm 0.20$ & $6.12 \pm 1.72^{* *}$ & $6.04 \pm 1.77$ & $6.18 \pm 1.82$ & $6.13 \pm 1.57$ & 0.766 \\
\hline $2 \mathrm{hPG}(\mathrm{mmol} / \mathrm{L})$ & $9.24 \pm 2.28$ & $16.78 \pm 3.75^{* *}$ & $16.39 \pm 3.95$ & $17.40 \pm 3.28$ & $16.54 \pm 3.98$ & 0.833 \\
\hline HbA1c (\%) & $\mathrm{N}$ & $9.46 \pm 2.29$ & $9.41 \pm 2.22$ & $9.91 \pm 2.54$ & $9.01 \pm 2.02$ & 0.346 \\
\hline IS $_{\text {HOMA- cp }}$ & $\mathrm{N}$ & $\begin{array}{l}10.06(2.72- \\
8.65)\end{array}$ & $\begin{array}{l}9.91(2.81- \\
9.16)\end{array}$ & $\begin{array}{l}12.86(2.72- \\
10.15)\end{array}$ & $\begin{array}{l}7.33(2.37- \\
7.64)\end{array}$ & 0.720 \\
\hline $\mathrm{UA}(\mu \mathrm{mol} / \mathrm{L})$ & $\mathrm{N}$ & $\begin{array}{l}314.36(250.0- \\
377.0)\end{array}$ & $\begin{array}{l}323.76(251.0- \\
404.0)\end{array}$ & $\begin{array}{l}309.7(248.3- \\
357.5)\end{array}$ & $\begin{array}{l}309.5(242.5- \\
380.5)\end{array}$ & 0.434 \\
\hline $\mathrm{TC}(\mathrm{mmol} / \mathrm{L} \square$ & $4.31 \pm 0.80$ & $4.44 \pm 0.82$ & $4.42 \pm 0.86$ & $4.61 \pm 0.86$ & $4.29 \pm 0.71$ & 0.377 \\
\hline $\mathrm{TG}(\mathrm{mmol} / \mathrm{L})$ & $\begin{array}{l}1.22(0.60- \\
1.54)\end{array}$ & $\begin{array}{l}2.11(1.32- \\
2.67)^{* *}\end{array}$ & $\begin{array}{l}2.45(1.58- \\
3.07)\end{array}$ & $\begin{array}{l}2.12(1.20- \\
2.74)\end{array}$ & $\begin{array}{l}1.76(1.18- \\
2.17)\end{array}$ & $0.001^{\# \# \#}$ \\
\hline HDL-c (mmol/L) & $1.34 \pm 0.17$ & $1.12 \pm 0.29 * *$ & $1.06 \pm 0.26$ & $1.13 \pm 0.23$ & $1.17 \pm 0.35$ & $0.025^{\#}$ \\
\hline LDL-c (mmol/L) & $2.54 \pm 0.56$ & $2.82 \pm 0.70 *$ & $2.82 \pm 0.70$ & $2.96 \pm 0.70$ & $2.67 \pm 0.67$ & 0.198 \\
\hline $\mathrm{LPa}(\mathrm{mg} / \mathrm{L})$ & $\mathrm{N}$ & $\begin{array}{l}186.5(45.0- \\
243.0)\end{array}$ & $\begin{array}{l}178.7(35.0- \\
264.0)\end{array}$ & $\begin{array}{l}171.7(45.0- \\
230.0)\end{array}$ & $\begin{array}{l}223.2(49.0- \\
245.0)\end{array}$ & 0.445 \\
\hline AIP & $-0.13 \pm 0.30$ & $0.23 \pm 0.25^{* *}$ & $0.33 \pm 0.23$ & $0.23 \pm 0.27$ & $0.15 \pm 0.21$ & 0.001 \#\#\# \\
\hline $\mathrm{baPWV}(\mathrm{cm} / \mathrm{s})$ & $\mathrm{N}$ & $1879.8 \pm 355.2$ & $2029.6 \pm 330.3$ & $1870.0 \pm 329.4$ & $1737.7 \pm 348.9$ & $0.001^{\# \# \#}$ \\
\hline
\end{tabular}

Categorical variables are frequency (percentage), normally distributed values in the table are mean \pm SD and nonnormally distributed values are median (25 and $75 \%$ interquartiles).

FGF19 fibroblast growth factor 19, BMI body mass index, SBP/DBP systolic/diastolic blood pressure, NAFLD nonalcoholic fatty liver disease, FPG fasting plasma glucose, 2hPG 2-hour postprandial blood glucose, HbA1c glycosylated

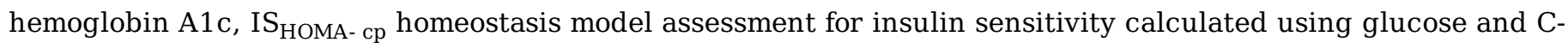
peptide in fasting status, UA uric acid, TC total cholesterol, TG triglyceride, HDL-c high density lipoprotein cholesterol, LDL-c low density lipoprotein cholesterol, LPa lipoprotein a, AIP atherogenic index of plasma, baPWV brachial-ankle pulse wave velocity, NFS NAFLD fibrosis score 
$\mathrm{p}$ values for continuous variables and categorical variables were determined by ANOVA and the Chi squared test, respectively. ${ }^{\#} \mathrm{p}<0.05,{ }^{\# \#} \mathrm{p}<0.01$, \#\# $\mathrm{p}<0.001$

${ }^{*} \mathrm{P}<0.05,{ }^{* *} \mathrm{P}<0.01$, the comparison of T2D with Controls

Table 2 Multiple linear regression models displaying adjusted estimates for lnFGF19 for outcomes of arteriosclerosis parameters adjusted for the other clinical covariates in each model in patients with T2D

\begin{tabular}{|c|c|c|c|c|c|}
\hline Models & B (95\% CI) & $\beta$ & $t$ & $p$ & $\begin{array}{l}R^{2} \text { for } \\
\text { model }\end{array}$ \\
\hline \multicolumn{6}{|l|}{ baPWV } \\
\hline Model 0: unadjusted & $\begin{array}{l}-167.6(-227.0 \\
\text { to }-108.3)\end{array}$ & $\begin{array}{c}- \\
0.37\end{array}$ & $\begin{array}{c}- \\
5.57\end{array}$ & $<0.001$ & 0.135 \\
\hline Model 1: age, sex, BMI and duration & $\begin{array}{l}-161.8(-218.7 \\
\text { to }-104.9)\end{array}$ & $\begin{array}{c}- \\
0.36\end{array}$ & - & $<0.001$ & 0.241 \\
\hline $\begin{array}{l}\text { Model 2: Model } 1 \text { + SBP, DBP, hypertension, statins, NAFLD, } \\
\text { NFS, UA, TC, TG, HDLC, LDLC, and Lpa }\end{array}$ & $\begin{array}{l}-98.7(-171.5 \text { to } \\
-26.0)\end{array}$ & $-\overline{2} 2$ & 2.70 & 0.008 & 0.587 \\
\hline $\begin{array}{l}\text { Model 3: Model } 2+\text { IS }_{\text {HOMA-cp }}, \mathrm{HbA1} \text { c and antidiabetic } \\
\text { treatments } \\
\text { lnAIP }\end{array}$ & $\begin{array}{l}-86.8(-164.5 \text { to } \\
-9.1)\end{array}$ & $-\overline{20}$ & $-\overline{2}$ & 0.029 & 0.599 \\
\hline Model 0: unadjusted & $\begin{array}{l}-0.22(-0.32 \text { to }- \\
0.12)\end{array}$ & $\begin{array}{c}- \\
0.30\end{array}$ & - & $<0.001$ & 0.091 \\
\hline Model 1: age, gender, BMI and diabetic duration & $\begin{array}{l}-0.21(-0.30 \text { to } \\
-0.11)\end{array}$ & $-\overline{2}$ & - & $<0.001$ & 0.145 \\
\hline $\begin{array}{l}\text { Model 2: Model } 1+\text { SBP, DBP, hypertension, statins, NAFLD, } \\
\text { NFS, UA, TC, LDLC and Lpa }\end{array}$ & $\begin{array}{l}-0.21(-0.35 \text { to }- \\
0.07)\end{array}$ & $-\overline{29}$ & $-\overline{0} \cdot$ & 0.003 & 0.342 \\
\hline $\begin{array}{l}\text { Model 3: Model } 2+\text { IS }_{\text {HOMA-cp }} \text { HbA1c and antidiabetic } \\
\text { treatments }\end{array}$ & $\begin{array}{c}-0.21(-0.36 \text { to }- \\
0.05)\end{array}$ & $-\overline{2}$ & $-\overline{-}$ & 0.010 & 0.491 \\
\hline
\end{tabular}

\section{Figures}


a.

unadjusted: $r=-0.351, p<0.001$

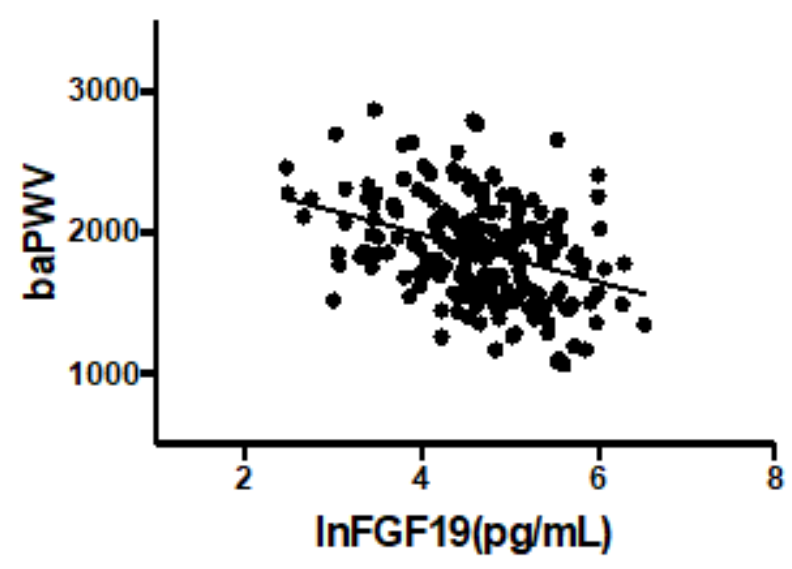

b.

partially adjusted: $r=-0.321, p<0.001$

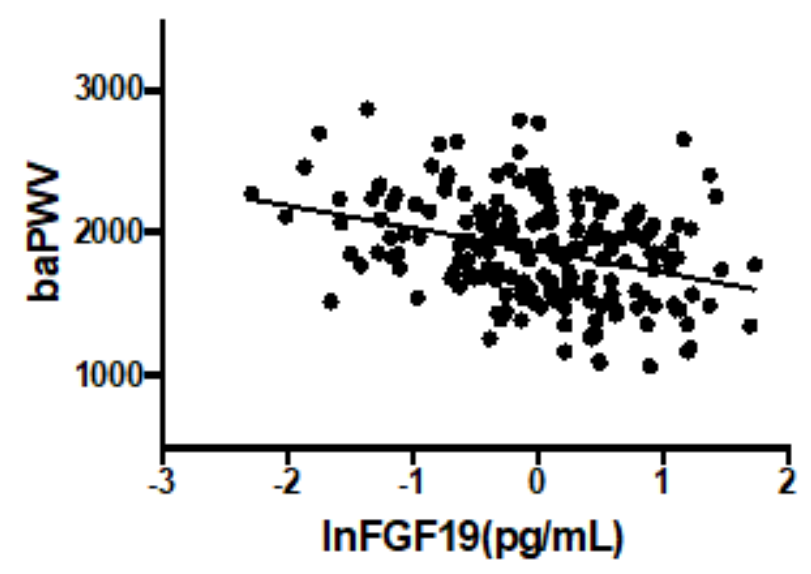

Figure 1

The relationship between InFGF19 and baPWV in patients with T2D (a unadjusted; b partially adjusted for age, gender, duration, BMI, hypertension, diabetic treatment, NAFLD) 
a.

unadjusted: $r=-0.303, p<0.001$

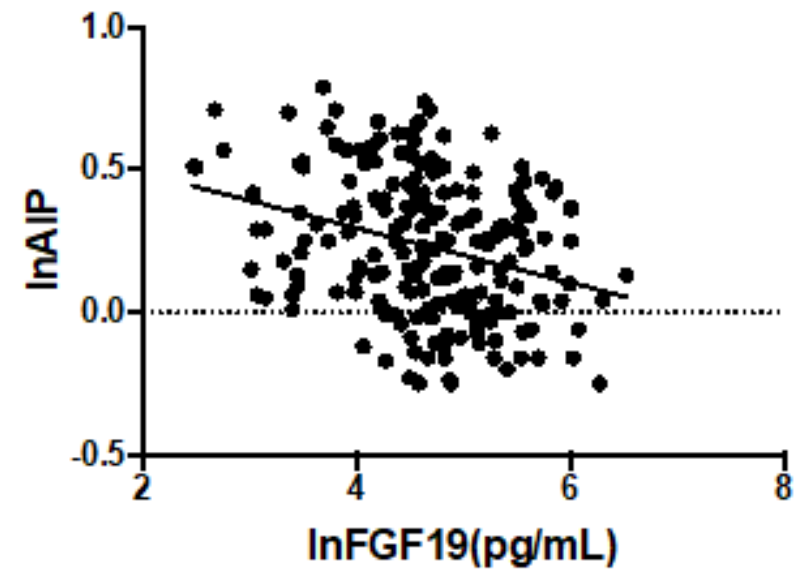

b.

partially adjusted: $r=-0.256, p<0.001$

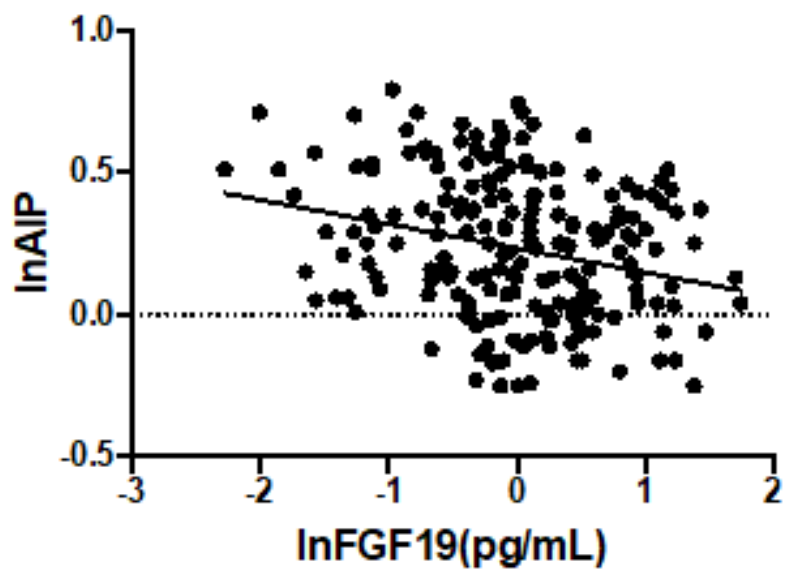

Figure 2

The relationship between InFGF19 and InAIP in patients with T2D (a unadjusted; b partially adjusted for age, gender, duration, BMI, hypertension, diabetic treatment, NAFLD) 